\title{
Search for $\beta>0.6$ magnetic monopoles with the ANTARES neutrino telescope
}

\author{
Imad El Bojaddaini* \\ Mohamed I University, Oujda, Morocco \\ E-mail: elbojaddaini_1990@hotmail.fr
}

\section{Antoine Kouchner ${ }^{\dagger}$}

APC, Univ Paris Diderot, France

E-mail: kouchnereapc.univ-paris7.fr

The search for magnetic monopoles with the ANTARES neutrino telescope is presented. These hypothetical particles carry only a magnetic charge. They would be created in the primordial Universe within the phase transition corresponding to the spontaneous breaking of the unified gauge group into subgroups, then would be accelerated by the Galactic magnetic fields and reach the Earth. Magnetic monopoles could be detected as highly ionizing particles in neutrino telescopes. This new analysis uses a total live time of 1121 days of the ANTARES detector. No signal above the background expectation from atmospheric muons and neutrinos is observed, and new 90\% C.L. upper limits are set on the magnetic monopole flux for velocities $\beta=v / c \geq 0.6$. These limits hold for up-going magnetic monopoles in the mass range $10^{10} \mathrm{GeV} / \mathrm{c}^{2} \leq M \leq 10^{14} \mathrm{GeV} / \mathrm{c}^{2}$.

35th International Cosmic Ray Conference ICRC2017

10-20 July, 2017

Bexco, Busan, Korea

\footnotetext{
* On behalf of the ANTARES collaboration

${ }^{\dagger}$ Speaker
} 


\section{Introduction}

The concept of electric monopole is very familiar in physics although it is known with its more common name "electric charge", because it exists in the form of particles that have positive or negative charges like electrons and protons. Opposite electric charges attract and like charges repel through the interaction of electric fields, which are defined as running from positive to negative. Magnetism seems analogous to electricity, as there exist a magnetic field with a direction defined as running from north to south. However, this analogy breaks down when trying to find the magnetic counterpart of the electric charge, the magnetic monopole (MM). This particle with only one magnetic pole has never been observed, instead, magnets exist only in the form of dipoles with a north and a south end.

The main theory introducing magnetic monopoles was presented by P. A. M. Dirac in 1931 [1]. Indeed, measured electric charges are always found to be integer multiples of the electron charge. This quantization of electric charge is a deep property of Nature without an explanation. Dirac discovered that the existence of magnetic monopoles explains the quantization of the electric charge in the framework of quantum mechanics.

In contrast to Dirac's demonstration of the consistency of magnetic monopoles with quantum mechanics, G. 't Hooft [2] and A. M. Polyakov [3] demonstrated independently in 1974 the necessity of magnetic monopoles in unified gauge theories. Any unified gauge theory in which the group U(1) describing electromagnetism is embedded in a spontaneously broken semisimple gauge group, and electric charge is thus automatically quantized, necessarily contains magnetic monopoles.

While there is no indication of the mass of the Dirac's magnetic monopole, in the context of GUTs the magnetic monopole mass $M$ is related to the mass of the $X$-boson carrier of the unified interaction $\left(m_{X} \sim 10^{15} \mathrm{GeV} / \mathrm{c}^{2}\right)$, yielding $M \gtrsim m_{X} / \alpha \simeq 10^{17} \mathrm{GeV} / \mathrm{c}^{2}$. An object this massive may have been produced only in the very early stages of the Universe after the Big Bang, and if the Universe cooled down to a point that MM creation was no longer energitically possible, perhaps MMs exist and the exponential expansion called inflation just distributed them everywhere. This was one of the motivations for the scenario of the inflationary Universe introduced by Guth [4] which explains the non-abundance of MMs. With the expansion of the Universe, the MM energy decreased, then MMs have been re-accelerated by the galactic magnetic fields. This acceleration process drains energy from the galactic magnetic field. An upper bound on the flux of MMs in the galaxy (called the Parker bound [5]) has been obtained by requiring the rate of this energy loss to be small compared to the time scale on which the galactic field can be regenerated.

Recent searches for magnetic monopoles created through electroweak interactions in the mass range $M<10 \mathrm{TeV}$ have been performed by the MoEDAL experiment at CERN. Since no candidates were found, upper limits were established on the MM production cross sections [6].

Neutrino and cosmic ray telescopes such as MACRO [7], Baikal [8], IceCube [9] and PierreAuger [10] attempted to detect magnetic monopoles with no positive result so far. The ANTARES Neutrino telescope has found upper limits on MM flux in a result published in [11].

In this paper, a new search for MMs is presented, using five years of the ANTARES detector data collected from 2008 to 2012, and corresponding to 1121 active days of live time. 


\section{Magnetic monopoles signature}

The Earth acts as a shield against all particles except neutrinos. The ANTARES neutrino telescope [12] uses the detection of up-going charged particles as a signature of neutrino interaction in the matter below the detector. The detection of muons in water through Cherenkov light emission allows the determination of their trajectory. This detection technique requires discriminating upgoing muons against the much higher flux of down-going atmospheric muons.

Since the ANTARES telescope is sensitive to up-going particles, this reduces the mass range of magnetic monopoles that can be observed. Indeed, the stopping power defined by Ahlen [13] has been used to estimate the energy loss of a monopole when crossing the Earth. However, despite the high energy loss, monopoles would remain relativistic and detectable as up-going events if their mass $M \gtrsim 10^{10} \mathrm{GeV} / c^{2}$. On the other hand, the monopole speed depends on the characteristics of the galactic magnetic fields, thus, given some astrophysical considerations, only monopoles with $M \lesssim 10^{14} \mathrm{GeV} / c^{2}$ are expected. The limits found in this analysis hold for monopoles with $10^{10} \mathrm{GeV} / c^{2} \lesssim M \lesssim 10^{14} \mathrm{GeV} / c^{2}$

The signature of a magnetic monopole in ANTARES would be similar to that of a high energy muon. MMs would induce the polarization of the medium to allow Cherenkov emission if their velocity exceeds the Cherenkov threshold $\beta_{t h}=1 / n \approx 0.74$. In addition, MMs can knock off atomic electrons that can have velocities above Cherenkov threshold, contributing to the total light yield by the so-called $\delta$-rays. The production of these $\delta$-electrons is described by the differential cross-section of Kasama, Yang and Goldhaber KYG [14] or the Mott cross section [15]. Fig. 1 shows the light yield with all these mechanisms compared to that from a minimum ionizing muon.

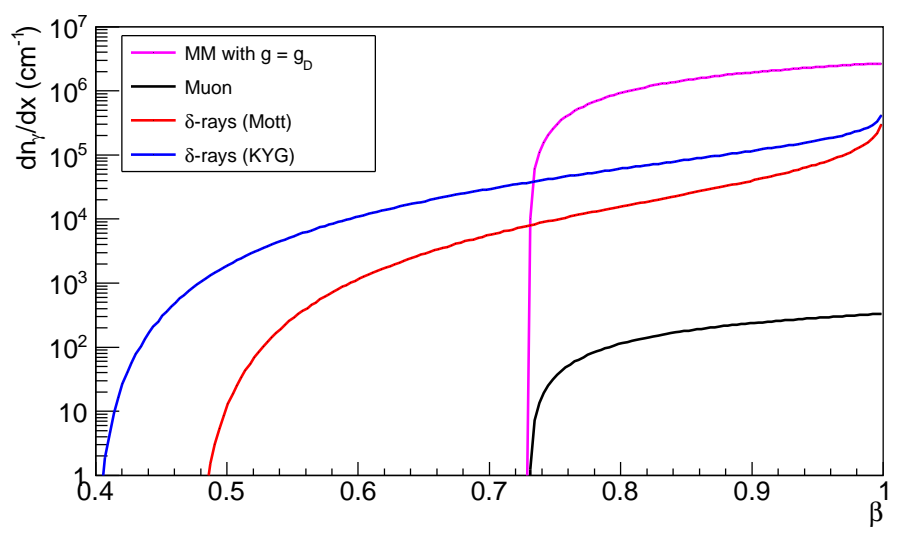

Figure 1: The total number of Cherenkov photons with wavelengths between 300 and $600 \mathrm{~nm}$ that are directly produced per centimeter path length by a MM with $g=g_{D}$, as a function of its velocity $(\beta)$. The number of photons produced by $\delta$-rays with Mott cross section model [15] and KYG cross section model [14] and by a minimum ionizing muon are also shown.

\section{Monte Carlo simulation and reconstruction}

MMs are simulated in nine ranges of velocity in the interval $\beta=[0.5945,0.9950]$ using a 
Monte Carlo program based on GEANT3. MM events are generated isotropically over the lower hemisphere of the detector. The propagation and detection of emitted photons is processed inside a virtual cylindrical surface surrounding the instrumented volume around the detector.

The simulation of atmospheric muons is carried out using the generator MUPAGE [16] based on the parametrisation of the angle and energy distributions of muons under-water as a function of the muon bundle multiplicity.

Up-going atmospheric neutrinos from the decay of pions and kaons are simulated using the package GENHEN [17] assuming the model from the Bartol group [18] which does not include the decay of charmed particles.

The analysis presented in this paper is based on a run-by run Monte Carlo strategy [19], which simulates each run of data individually taking into consideration its actual conditions (e.g. sea water conditions, bioluminescence variability, detector status).

The reconstruction algorithm [20] performs two independent fits: a track fit and a bright-point fit. The former reconstructs particles crossing the detector, while the latter reconstructs showering events, as those induced by the charged current interactions. Both fits minimize the same $\chi^{2}$ quality function, thus, two parameters defining the quality of these reconstructions are introduced, $t \chi^{2}$ for the track fit, and $b \chi^{2}$ for the bright-point fit. Fig. 2 shows the distribution of $t \chi^{2}$ for atmospheric muons and neutrinos. The search strategy was based on a blind analysis, such as the selection cuts applied are established on Monte Carlo simulation and using a test data sample of about $10 \%$ of the total data set. The neutrino distribution represents electron and muon neutrinos for both neutral and charged currents. In order to select data taken in good conditions, some basic quality cuts have been applied.

Event reconstruction has been performed assuming the reconstructed velocity of the particle $\beta_{\text {rec }}$ as a free parameter to be derived by the track fit. This improves the sensitivity for monopoles travelling with $\beta \leq 0.81$.

\section{Event selection and optimization}

In order to get rid of the bulk of down-going background events, only up-going events are selected. From Fig. 2, one can notice the significant agreement between the simulated atmospheric background and the sample of data. This was achieved by applying the cut $t \chi^{2} \leq b \chi^{2}$ in order to favor the reconstructed tracks rather than showers.

Despite quality cuts, the selected event sample remains dominated by atmospheric muons for low velocities. Additional cuts on the track fit quality parameter are implemented to remove misreconstructed atmospheric muon tracks. The selection of the events was further optimized for different MM velocities. A different event selection was performed for each of the nine bins of $\beta$.

The hits from the optical modules belonging to the same storey are summed together to form a track hit. For all velocity bins, the number of storeys with selected track hits $N_{h i t}$ is used as a powerfull discriminant variable since it refers to the amount of light emitted. A second discriminative variable is introduced to further reduce the background in particular for lower velocities where the light emission is less. This variable named $\alpha$ is defined from a combination of the track fit quality parameter and $N_{h i t}$, and allows to avoid that bright events get cut by the condition applied on the $t \chi^{2}$ variable. Fig. 3 shows the distribution of $\alpha$ and $N_{h i t}$ used in the optimization. 


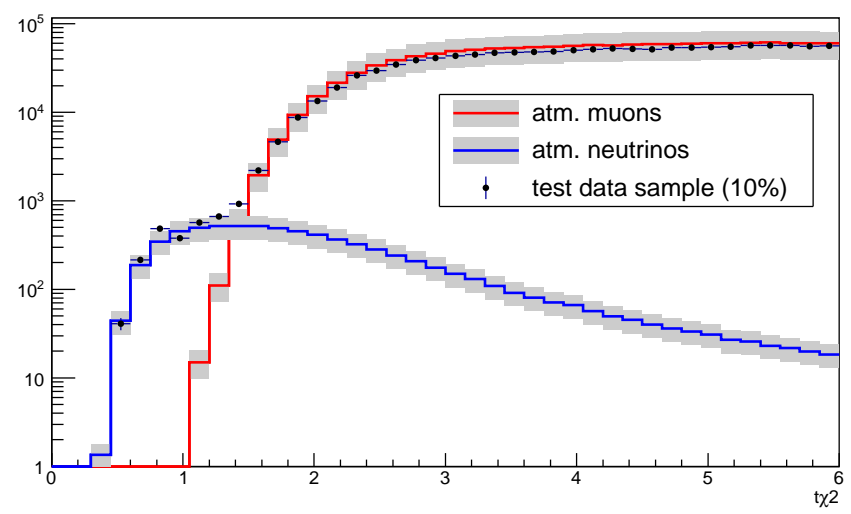

Figure 2: The distribution of $t \chi^{2}$ for atmospheric muons, atmospheric neutrinos (red and blue histograms, respectively) and data (points with error bars). The systematic uncertainties on the flux of atmospheric muons and neutrinos are represented by error bands. All distributions correspond to events reconstructed as up-going, and the cut $t \chi^{2} \leq b \chi^{2}$ has been applied as it allows to choose tracks rather than showers.

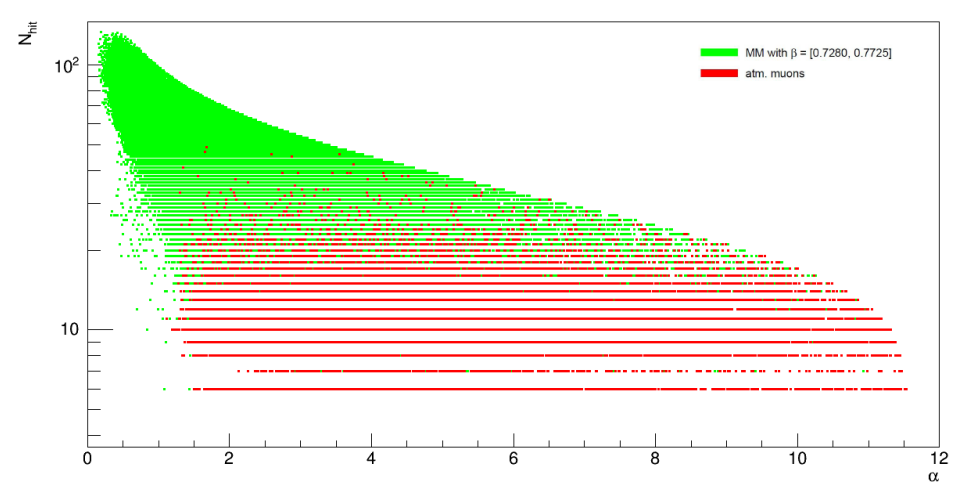

Figure 3: Two-dimensional distribution of $\alpha$ and $N_{h i t}$, for atmospheric muons (red) and MMs (green) simulated in the velocity range $[0.7280,0.7725]$. The distributions correspond to up-going events with $t \chi^{2} \leq b \chi^{2}$ and $\beta_{\text {rec }}=[0.7280,0.7725]$. No neutrinos survived at this range of $\beta$.

\section{Uncertainties}

Taking into account the statistical uncertainties, an extrapolation of $N_{h i t}$ distribution is performed for atmospheric muons in order to compensate the lack of statistics. After fitting the $N_{\text {hit }}$ distribution with a Landau type function, the latter is extrapolated to the region of interest for the signal (see Fig. 4). The number of muons remaining after the final cut on $N_{h i t}$ is given by the sum of the events remaining from the muon histogram and those remaining from the extrapolated function.

The contribution of atmospheric neutrinos in the calculation of the upper limits is negligible compared to that from atmospheric muons (see Table 1 in the next section). Thus the effect on the neutrino rate due to the detector uncertainties are not considered. Concerning the atmospheric muons, the dominant effect is due to the lack of statistics as described in Fig. 4. It is larger than 


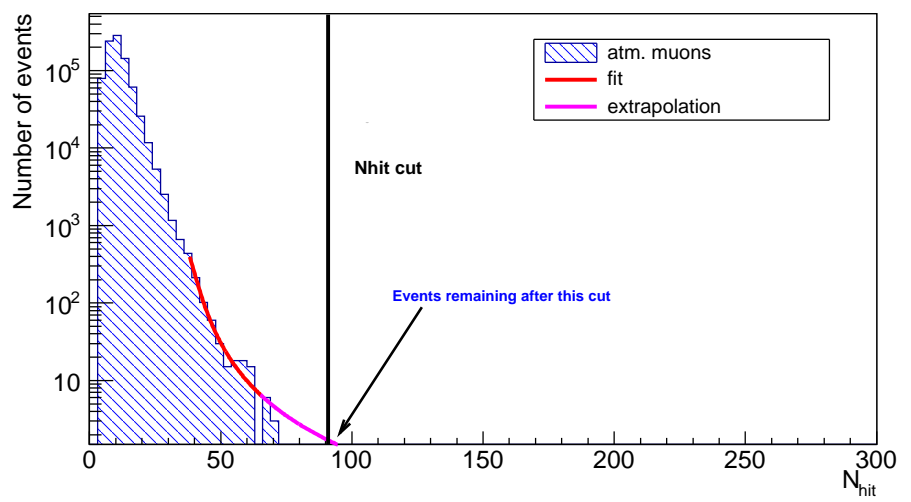

Figure 4: The distribution of $N_{\text {hit }}$ for atmospheric muons, extrapolated using a Landau fit function. The contribution of the extrapolation in the total number of events was taken into account in the optimization and the extrapolation uncertainties were computed. For this bin $\beta=[0.8170,0.8615], 1.4$ events are found after the cut $N_{\text {hit }} \geqslant 91$.

$50 \%$ in most cases (see column 3 of Table 1), while the uncertainty on the optical module and the uncertainty on the light absoption and scattering lengths in water yield an overall effect of $35 \%$ for muons and 30\% for neutrinos [21]. These uncertainties are represented in Fig. 2 by an error band around each histogram.

\section{Results}

Using the Feldman-Cousins approach [22], the selection cuts are optimized by minimizing the so-called Model Rejection Factor (MRF) [23].

After applying the unblinding on the total set of data collected by ANTARES from 2008 to 2012 that corresponds to 1012 active days live time after extracting the $10 \%$ data sample, no significant excess is observed over the atmospheric background expectation and upper limits on MMs flux at 90\% C.L. are found and quoted in Table 1, for each bin of $\beta$.

In the first five bins, the reconstructed velocity $\beta_{\text {rec }}$ was restricted to be compatible with the range of the MM velocity. In the last bins, $\beta$ was not reconstructed as it does not contribute to isolate the MM signal.

Fig. 5 represents the ANTARES upper limits found as a function of MM $\beta$, and compared to different experiments such as IceCube [9], MACRO [7] and Baikal [8], as well as the previous result from ANTARES [11] and the theoretical Parker bound [5].

\section{Conclusion}

This paper presented the ANTARES upper limits found with five years of data recorded between 2008 and 2012 and corresponding to 1012 days of live time. This new analysis was based on a Monte Carlo strategy which consists in simulating each run individually, in order to take into account its specific conditions ("run-by-run" simulation). 


\begin{tabular}{|c|cc|c|c|c|c|}
\hline$\beta$ range & \multicolumn{2}{|c|}{$\begin{array}{c}\text { Selection cuts } \\
\alpha\end{array} N_{\text {hit }}$} & $\begin{array}{c}\text { Number of } \\
\text { atm. muons }\end{array}$ & $\begin{array}{c}\text { Number of } \\
\text { atm. neutrinos }\end{array}$ & $\begin{array}{c}\text { Number of } \\
\text { obs. events }\end{array}$ & $\begin{array}{c}\text { Flux Upper Limits } \\
\text { 90\% C.L. }\left(\mathrm{cm}^{-2} \cdot \mathrm{s}^{-1} \cdot \mathrm{sr}^{-1}\right)\end{array}$ \\
\hline \hline$[0.5945,0.6390]$ & $<5.5 \geqslant 36$ & $1.9 \pm 0.8$ & $1.6 \times 10^{-4}$ & 0 & $5.9 \times 10^{-16}$ \\
\hline$[0.6390,0.6835]$ & $<5.0 \geqslant 39$ & $0.9 \pm 0.5$ & $1.5 \times 10^{-4}$ & 0 & $3.6 \times 10^{-17}$ \\
\hline$[0.6835,0.7280]$ & $<3.4 \geqslant 51$ & $0.9 \pm 1.0$ & $1.2 \times 10^{-4}$ & 0 & $2.1 \times 10^{-17}$ \\
\hline$[0.7280,0.7725]$ & $<3.3 \geqslant 51$ & $1.1 \pm 0.5$ & $9.3 \times 10^{-3}$ & 1 & $9.1 \times 10^{-18}$ \\
\hline$[0.7725,0.8170]$ & $<1.8 \geqslant 73$ & $0.6 \pm 0.4$ & $1.0 \times 10^{-3}$ & 0 & $4.5 \times 10^{-18}$ \\
\hline$[0.8170,0.8615]$ & $<0.8 \geqslant 91$ & $1.4 \pm 0.9$ & $1.8 \times 10^{-1}$ & 1 & $4.9 \times 10^{-18}$ \\
\hline$[0.8615,0.9060]$ & $<0.6 \geqslant 92$ & $1.3 \pm 0.8$ & $1.6 \times 10^{-1}$ & & $2.5 \times 10^{-18}$ \\
\hline$[0.9060,0.9505]$ & $<0.6 \geqslant 94$ & $1.2 \pm 0.8$ & $1.3 \times 10^{-1}$ & 0 & $1.8 \times 10^{-18}$ \\
\hline$[0.9505,0.9950]$ & $<0.6 \geqslant 95$ & $1.2 \pm 0.7$ & $1.3 \times 10^{-1}$ & 0 & $1.5 \times 10^{-18}$ \\
\hline
\end{tabular}

Table 1: Results after unblinding of the data (1012 active days live time corresponding to 5 years of data taking). The selection cuts, the number of expected (muons and neutrinos) background and observed events and the upper limits on the flux are presented for each range of velocity $(\beta)$. The table was divided into two parts to distinguish the first five bins where $\beta_{\text {rec }}$ was assumed as a free parameter from the four bins where $\beta_{\text {rec }}=1$.

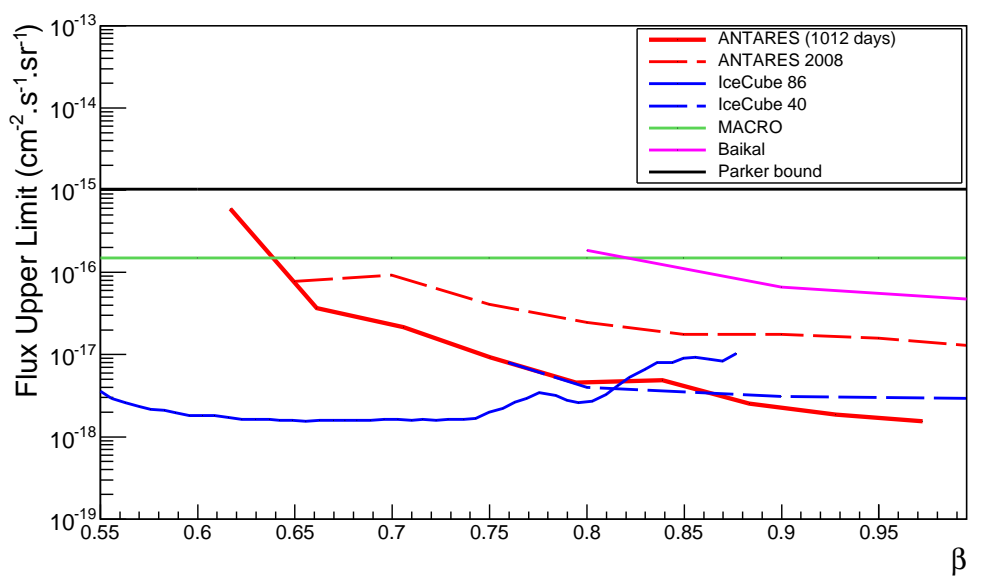

Figure 5: ANTARES 90\% C.L. upper limit on flux for MMs using five years of data with 1012 active days live time (solid red line), compared to the upper limits obtained by other experiments $[9,7,8]$, as well as the previous analysis of ANTARES (dashed red line) [11] and the theoretical Parker bound [5]. In [9] a more optimistic model for $\delta$-rays production of MMs is used, making a direct comparison difficult.

The limits presented show a good result at high velocities. Below the Cherenkov threshold $\beta=0.74$, the model of cross section for interactions between monopoles and electrons used in this analysis is the Mott model [15], which provides less light compared to the KYG model [14] used by IceCube, making a direct comparison between the two results difficult. The use of the Mott cross section allows a simpler application in time-consuming Monte Carlo simulations of the energy spectrum of the produced $\delta$-electrons. Additionally, the Mott prediction yields a safer and conservative estimate of the total light yield. 
This analysis opens a new window to the search for magnetic monopoles using the future detector KM3NeT [24] that will certainly improve the sensitivity to their detection due to its large volume and high detection performance.

\section{References}

[1] P.A.M. Dirac, Proc. R. Soc. A 133 (1931) 60.

[2] G. 't Hooft, Nucl. Phys. B 79 (1974) 276.

[3] A.M. Polyakov, Sov. Phys. JETP Lett. 20 (1974) 194.

[4] A.H. Guth, Phys. Rev. D 23 (1981) 347.

[5] E.N. Parker, Astrophys. J 160 (1970) 383.

[6] B. Acharya et al., JHEP 08 (2016) 067.

[7] M. Ambrosio et al., Eur. Phys. J. C 25 (2002) 511.

[8] V. Aynutdinov et al., Astropart. Phys. 29 (2008) 366.

[9] M.G. Aartsen et al., EPJ C76 (2016) 133.

[10] A. Aab et al., Phys.Rev. D94 (2016) 082002.

[11] S. Adrian-Martinez et al., Astropart. Phys. 35 (2012) 634.

[12] M. Ageron et al., Nucl. Instr. and Meth. A 656 (2011) 11.

[13] S.P. Ahlen, Phys. Rev. D 17, 1 (1978) 229.

[14] Y. Kazama et al., Phys. Rev. D 15 (1977) 2287.

[15] S.P. Ahlen, Phys. Rev. D 14 (1976) 2935.

[16] G. Carminati et al., Comput. Phys. Commun. 179(12) (2008) 915.

[17] A. Margiotta, NIM A725 (2013) 98.

[18] G.D. Barr et al., Phys. Rev. D 74 (2006) 094009.

[19] L. Fusco and A. Margiotta, EPJ Web of Conferences 116 (2016) 02002.

[20] J.A. Aguilar et al., Astropart. Phys. 34 (2011) 652.

[21] J.A. Aguilar et al., Astropart. Phys. 34 (2010) 179.

[22] G.J. Feldman and R.D. Cousins, Phys. Rev. D 57 (1998) 3873.

[23] G.C. Hill and K. Rawlins, Astropart. Phys. 19 (2003) 393.

[24] S. Adrian-Martinez et al., J. Phys. G43(8) (2016) 084001. 